\title{
Deforming the Maxwell-Sim algebra
}

\author{
G. W. Gibbons, ${ }^{1}$ Joaquim Gomis, ${ }^{2}$ and C. N. Pope ${ }^{3,1}$ \\ ${ }^{1}$ DAMTP, Centre for Mathematical Sciences, Cambridge University, Wilberforce Road, Cambridge CB3 OWA, United Kingdom \\ ${ }^{2}$ Departament d'Estructura i Constituents de la Matèria and ICCUB, Universitat de Barcelona, \\ Diagonal 647, 08028 Barcelona, Spain \\ ${ }^{3}$ George and Cynthia Woods Mitchell Institute for Fundamental Physics and Astronomy, Texas A\&M University, \\ College Station, Texas 77843-4242, USA
}

(Received 25 May 2010; published 1 September 2010)

The Maxwell algebra is a noncentral extension of the Poincaré algebra, in which the momentum generators no longer commute, but satisfy $\left[P_{\mu}, P_{\nu}\right]=Z_{\mu \nu}$. The charges $Z_{\mu \nu}$ commute with the momenta, and transform tensorially under the action of the angular momentum generators. If one constructs an action for a massive particle, invariant under these symmetries, one finds that it satisfies the equations of motion of a charged particle interacting with a constant electromagnetic field via the Lorentz force. In this paper, we explore the analogous constructions where one starts instead with the ISim subalgebra of Poincaré, this being the symmetry algebra of very special relativity. It admits an analogous noncentral extension, and we find that a particle action invariant under this Maxwell-Sim algebra again describes a particle subject to the ordinary Lorentz force. One can also deform the ISim algebra to DISim ${ }_{b}$, where $b$ is a nontrivial dimensionless parameter. We find that the motion described by an action invariant under the corresponding Maxwell-DISim algebra is that of a particle interacting via a Finslerian modification of the Lorentz force. In an appendix is it shown that the $\operatorname{DISim}_{b}$ algebra is isomorphic to the extended Schrödinger algebra with its standard deformation parameter $z$, when $b=\frac{1}{1-z}$.

DOI: 10.1103/PhysRevD.82.065002

PACS numbers: 02.20.Sv, 11.10.Ef, 11.30.Cp

\section{INTRODUCTION}

A popular line of thought in theoretical physics is to start with a Lie algebra $g$ or Lie group $G$, and then to construct from it the space or spacetime in which physical objects, for example, $p$-branes, move. Typically the spaces or spacetimes are cosets $G / H$. The dynamics of $p$-branes is then described as a map from the $(p+1)$-dimensional world volume into $G / H$ [1-5]. In the case of point particles, the dynamics is often thought of as geodesic motion, or some modification thereof by "forces," such as the Lorentz force on electrically charges particles in electromagnetism, with respect to a metric on $G / H$ that is invariant under the left action of $G$ on $G / H$. More generally, one is interested in invariant Lagrangians $L(x, v)$ on the tangent space $T(G / H)$, or Hamiltonians $H(x, p)$ on the cotangent space $T^{\star}(G / H)$. For a recent statement of this viewpoint in the context of quantum field theory, see [6].

An alternative construction of a $p$-brane action in the space $G / H$ is to consider the quotient $(G / H) / K$, where $K$ is the stabilizer of the $p$-brane. The action of lowest order in derivatives is obtained by considering the pullback to the world volume of a ( $p+1)$-form invariant under $K$ [7] (see also [8], where one can find further references). The action contains extra Goldstone fields associated with the broken "rotations." In order to make contact with the geometrical Lagrangian $L(x, v)$, we should eliminate the extra fields by their nondynamical equations of motion or, more generally, by the inverse Higgs mechanism [9].

An early example of this program followed the discovery of the three congruence geometries: hyperbolic or
Lobachevsky space $H^{3}$, Euclidean space $\mathbb{E}^{3}$, and spherical space $S^{3}$. Helmholtz characterized these three possibilities physically in terms of axioms of the free mobility of rigid bodies [10]. Such bodies permit rotations about any point in space, and translations to any point in space. Thus he demanded that $H=S O(3)$ and that $G$ act transitively on $G / H$. He arrived, after some additional arguments, at the three possibilities

$$
\begin{gathered}
G=S O(3,1): G / H=H^{3}, \\
G=E(3): G / H=\mathbb{E}^{3}, \\
G=S O(4): G / H=S^{3} .
\end{gathered}
$$

An equivalent way of looking at this is to say that the configuration space $Q$ of a rigid body with one point fixed admits a simply transitive left action by $S O(3)$, and may thus be identified with $S O(3)$. Free motion of a rigid body is given by geodesic motion on $S O(3)$ with respect to a leftinvariant metric. If the body moves in ordinary Euclidean space, $Q$ is enlarged to become the Euclidean group $E(3)$. If the body moves in an inviscid fluid, conservation of momentum and angular momentum will still hold and the metric is then given by a general left-invariant metric on $E(3)$. Correspondingly, geodesic motion on $S O(3,1)$ or $S O(4)$ with respect to a left-invariant metric gives the motion of a rigid body moving in a fluid in $H^{3}$ or $S^{3}$, respectively.

A slightly different strand of thought begins with the observation (originally due to Lambert [11]) that passing to 
$S^{3}$ or $H^{3}$ introduces a new parameter into physics: the radius of curvature. This new parameter is associated with the fact that the translations in $S O(3,1)$ or $S O(4)$ no longer commute. Expressed mathematically, the Lie algebras $\mathfrak{S D}(3,1)$ and $\mathfrak{S D}(4)$ are continuous deformations of $\mathfrak{e}(3)$, and this suggests that in seeking new physical laws a fruitful procedure is to look for continuous deformations of existing laws. In algebraic terms, this translates into looking for continuous deformations of the Lie algebra $g$ that one begins with. Given that one has introduced a new physical parameter whose magnitude is arbitrary, it is natural to enquire whether it might be time-dependent. In the case of spatial curvature, just such a suggestion was made by Calinon long before general relativity and the Robertson-Walker metric [12].

The group theory viewpoint came into its own with Einstein's theory of special relativity, for which $G=$ $E(3,1)=I S O(3,1)$, the Poincaré group. Indeed only retrospectively was the Galilei group recognized as its Wigner-İnönü [13] contraction. As with the Euclidean group, the Poincaré group admits two continuous deformations, to $S O(4,1)$ or $S O(3,2)$, for which spacetime translations fail to commute. It was perhaps only the early death of Minkowski which delayed until after the advent of Einstein's general relativity the implementation of Calinon's idea. de Sitter, seeking a covariant version of Einstein's static universe, introduced their cosets, de Sitter and anti-de Sitter, respectively.

Einstein did not scruple to break boost invariance with his static universe [14], and this is a feature of all Robertson-Walker metrics except those of de Sitter [15]. A natural question, answered by Bacry and Levy-Leblond [16], is what other ten-dimensional kinematical algebras exist, which contain rotations, translations in time and space, and boosts. All can be regarded as Wigner-İnönü contractions of the de Sitter and anti-de Sitter algebras.

Invariance under the local Lorentz group is extremely well attested by experiment, but nevertheless Cohen and Glashow [17] observed that if it is broken down to its fourdimensional maximal subgroup $\operatorname{Sim}(2) \subset S O(3,1)$ it leaves invariant no spurion fields, merely leaving fixed a null direction $n^{\nu} \equiv \lambda n^{\nu}, \lambda \neq 0$, where $\eta_{\mu \nu} n^{\mu} n^{\nu}=0$. It may play a role linking small neutrino masses, and is compatible with all present-day tests of violations of Lorentz invariance. Thus they proposed in their very special relativity theory that the fundamental local symmetry group is the semidirect product of $\operatorname{Sim}(2)$ and the translations, known as $\operatorname{ISim}(2) \subset I S O(3,1)$. In recent work, in an attempt to obtain noncommuting translations, and hence spacetime curvature [18], we studied the continuous deformations of ISim(2) and found a two-parameter family, one of which was rejected because the deformation of the $S O(2)$ rotation generator ceased to be compact. The remaining one-parameter deformed group $\operatorname{DISim}(2)_{b}$ depends on a dimensionless parameter $b$, and coincides with one introduced by Bogoslovsky [19] in his proposal for an anisotropic Finslerian spacetime.

It is straightforward to generalize the $\operatorname{DIsim}(2)_{b}$ group to $k+2$ spacetime dimensions. We denote the resulting group $\operatorname{DISim}_{b}(k)$. It is interesting to note [20] that the $\operatorname{DISim}_{b}(k)$ is then isomorphic to the extended Schrödinger group $\widetilde{\operatorname{Sch}}(k)$ [21] which has resurfaced in recent studies of nonrelativistic holography in $k$ spatial dimensions (see, e.g., [22] and references therein). Since this topic is not strictly connected with the Maxwell algebra which is the main concern of the present paper, we relegate the details to Appendix B.

Since Maxwell's equations are invariant under $\operatorname{DISim}(2)_{b}$, the dispersion relation for photons is the standard one, and hence these theories are consistent with the recent high-precision test of Lorentz violation using the gamma-ray burst GRB090510 [23].

The advent of quantum mechanics led to the realization that not only are deformations of algebras important, but so also are extensions, especially central extensions. The Ur-example is the Heisenberg algebra

$$
\left[\hat{q}^{j}, \hat{p}_{i}\right]=i \hbar \delta_{i}^{j}
$$

However, a more relevant example for our purposes is the motion of a particle of charge $e$ in a uniform timeindependent magnetic field. The minimally coupled Lagrangian is

$$
L=T+e A_{i} \dot{x}^{i},
$$

where $A_{i}=-\frac{1}{2} F_{i j} x^{j}$, and $T$ is the kinetic energy. If $p_{i} \equiv$ $\partial T / \partial \dot{x}^{i}$ (sometimes called the mechanical momentum), then the canonical momentum is

$$
\pi_{i} \equiv \frac{\partial L}{\partial \dot{x}^{i}}=p_{i}+e A_{i}=p_{i}-\frac{1}{2} e F_{i j} x^{j} .
$$

Assuming that $\pi_{i}$ and $x^{j}$ satisfy the standard Poisson algebra $\left\{x^{i}, \pi_{j}\right\}=\delta_{j}^{i},\left\{x^{i}, x^{j}\right\}=\left\{\pi_{i}, \pi_{j}\right\}=0$, then $p_{i}$ and $x^{j}$ satisfy the centrally extended algebra

$$
\left\{x^{i}, p_{j}\right\}=\delta_{j}^{i}, \quad\left\{x^{i}, x^{j}\right\}=0, \quad\left\{p_{i}, p_{j}\right\}=e F_{i j} .
$$

The action associated to (1.5) is invariant, up to a boundary term, under constant translations:

$$
x^{i} \rightarrow x^{i}+a^{i} .
$$

The Noether charges or moment maps associated with these spatial translations are $a^{i} \tilde{p}_{i}$, where

$$
\tilde{p}_{i}=\pi_{i}-\frac{1}{2} e F_{i j} x^{j}=p_{i}-e F_{i j} x^{j} .
$$

It follows from the equations of motion that $d \tilde{p}_{i} / d t=0$. One finds the following nontrivial Poisson brackets:

$$
\left\{\tilde{p}_{i}, \tilde{p}_{j}\right\}=-e F_{i j}, \quad\left\{x^{i}, \tilde{p}_{j}\right\}=\delta_{j}^{i} .
$$


Note that the $\tilde{p}_{i}$ and $p_{i}$ momenta Poisson commute:

$$
\left\{\tilde{p}_{i}, p_{j}\right\}=0 .
$$

The constancy of the $\tilde{p}_{i}$ is now seen to follow from the fact that the Hamiltonian obtained by taking the Legendre transform of the Lagrangian (1.5) is a function only of the mechanical momentum $p_{i}$, and independent of the spatial coordinates $x^{i}$.

One can think of $e$ as a central element $-\bar{Z}$ in a finitedimensional Poisson algebra, which commutes with all other generators. The moment maps $p_{i}$ and $\bar{Z}$ generate a group of transformations on phase space, whose Lie algebra is

$$
\left[\mathbf{P}_{i}, \mathbf{P}_{j}\right]=\mathbf{Z} F_{i j}, \quad\left[\mathbf{P}_{i}, \mathbf{Z}\right]=0 .
$$

(The relative sign between Lie algebra brackets and Poisson brackets is a consequence of our general conventions, which are detailed in Appendix A.) Note that whereas the "central term" in the Poisson algebra (1.7) is cohomologically trivial (it can be removed by the local redefinition of generators that maps from $x^{i}$ and $p_{i}$ to $x^{i}$ and $\pi_{i}$ ), the central term in the Lie algebra (1.12) is cohomologically nontrivial. The reason for the difference is that the $x^{i}$ are included as generators in the Poisson algebra, but not in the Lie algebra.

Inclusion of a uniform time-independent electric as well as a magnetic field generalizes (1.12) to the Lorentzcovariant Lie algebra

$$
\left[\mathbf{P}_{\mu}, \mathbf{P}_{\nu}\right]=\mathbf{Z} F_{\mu \nu} .
$$

This five-dimensional algebra has as group manifold $G$, the five-dimensional space with coordinates $x^{\mu}$ and $\theta$, conjugate to $\mathbf{P}_{\mu}$ and $\mathbf{Z}$, respectively. It turns out, in Kaluza-Klein fashion, that geodesic motion on $G$ projects down onto the coset $G / H$, where $H \equiv \mathbb{R}$ is generated by the central element $\mathbf{Z}$, and electric charge is the conserved momentum in that direction. Particles with magnetic as well as electric charge (dyons) may also be catered for, by passing to six dimensions and replacing (1.13) by

$$
\left[\mathbf{P}_{\mu}, \mathbf{P}_{\nu}\right]=\mathbf{Z} F_{\mu \nu}+\mathbf{Z}^{\star} F_{\mu \nu}^{\star}
$$

where $F_{\mu \nu}^{\star}=\frac{1}{2} \epsilon_{\mu \nu \alpha \beta} F^{\alpha \beta}$ is the Hodge dual of $F_{\mu \nu}$.

A different approach is to consider noncentral extensions of the fundamental algebra $\mathrm{g}$. This is by now standard in supersymmetric $p$-brane theories, following the pioneering work of van Holten and van Proeyen [24]. However, the simplest example, which is purely bosonic and predates their work, is the 16-dimensional Maxwell algebra, which is a noncentral extension of the Poincaré algebra with six tensorial charges $\mathbf{Z}_{\mu \nu}$ arising through a noncommutativity of the momentum generators,

$$
\left[\mathbf{P}_{\mu}, \mathbf{P}_{\nu}\right]=\mathbf{Z}_{\mu \nu}
$$

One now introduces six angles conjugate to $\mathbf{Z}_{\mu \nu}$. These angles are dynamical variables with a nontrivial evolution.
Note that for any particular solution of the relevant equations of motion, $\bar{Z}_{\mu \nu}=-e F_{\mu \nu}$, spontaneous symmetry breaking will occur and the symmetry will be reduced to the subgroup of the Poincare group leaving the background $F_{\mu \nu}$ invariant. This is the kinematical group in this context.

The aim of this paper is to study in the framework of the very special relativity [17], or its deformation [18], the motion of a bosonic charged massive particle in the presence of a constant electromagnetic field. This will be done by constructing the noncentral extensions and deformations of ISim(2). As we shall see, the Maxwell-Sim algebra is constructed from the translation generators $\mathbf{P}_{\mu}$ and the noncentral extension $\mathbf{Z}_{\mu \nu}=\left[\mathbf{P}_{\mu}, \mathbf{P}_{\nu}\right]$, together with the $\operatorname{Sim}(n)$ generators $\left(\mathbf{M}_{+i}, \mathbf{M}_{+-}, \mathbf{M}_{i j}\right)$.

Later we study the deformations of the Maxwell-Sim algebra. In general dimensions we find two deformations, with parameters $b$ and $c$. The deformation parametrized by $c$ is analogous to the $k$ deformation of the Maxwell algebra found in [25] [now restricted to the 14 generators of Maxwell-Sim(2)], which gave $S O(3,2) \times S O(3,1)$ or $S O(4,1) \times S O(3,1)$, depending on the sign of $k$. The $b$ deformation of Maxwell-Sim produces the Maxwell extension of the $\operatorname{DISim}_{b}$ algebra, which is related to Finslerian geometry.

In order to construct the particle models with the previous symmetries, we use two different approaches: one based in the Lagrangian formalism and the nonlinear realization approach [26], and the other based on the Hamiltonian formalism constructed from the momentum maps.

In the case of Maxwell-DISim ${ }_{b}$ the motion is given by a Finslerian Lorentz force, while for the undeformed Maxwell-Sim we obtain the ordinary Lorentz force. Therefore the study of anisotropies of a massive particle in an electromagnetic field could provide a test of a possible Finsler geometry.

The organization of the paper is as follows. In Sec. II we review the Maxwell algebra, and in Sec. III we recall the basic facts about the ISim algebra. In Sec. IV we construct the Maxwell-Sim algebra, and then in Sec. V we the study its deformations. The particle Lagrangians are constructed in Sec. VI, and in Sec. VII we perform the Hamiltonian analysis. The paper ends with conclusions. Appendix A discusses the Hamiltonian formalism and moment maps, and Appendix B discusses the Lifshitz and Schrödinger algebras.

\section{THE MAXWELL ALGEBRA}

The name Maxwell algebra appears to originate with Glashow, as reported in [27] in connection with the behavior of matter in extremely strong magnetic fields such as are found in neutron stars. Nowadays one might think of magnetars. However, it is Schrader [28] who seems to have been the first to study it systematically. Other earlier work applying group theoretic methods to uniform electromagnetic 
fields is in $[29,30]$. This often-cited work assumes a constant $c$-number background field $F_{\mu \nu}$, and is largely concerned with what they called the kinematical group, i.e., with the six-dimensional subgroup of the Poincare group that leaves $F_{\mu \nu}$ invariant, generated by $\mathbf{P}^{\mu}$ and two commuting Lorentz generators

$$
\mathbf{G}=\frac{1}{2} F^{\mu \nu} \mathbf{M}_{\mu \nu}, \quad \mathbf{G}^{\star}=\frac{1}{2} F^{\star \mu \nu} \mathbf{M}_{\mu \nu} .
$$

This gives the algebra

$$
\begin{gathered}
{\left[\mathbf{G}, \mathbf{G}^{\star}\right]=0,} \\
{\left[\mathbf{G}, \mathbf{P}_{\mu}\right]=F_{\mu \nu} \mathbf{P}^{\nu},} \\
{\left[\mathbf{G}^{\star}, \mathbf{P}_{\mu}\right]=F^{\star}{ }_{\mu \nu} \mathbf{P}^{\nu},}
\end{gathered}
$$

where we have defined $F^{\star}{ }_{\mu \nu}=\frac{1}{2} \epsilon_{\mu \nu \rho \sigma} F^{\rho \sigma}$. We shall refer to these six-dimensional kinematical algebras as the BacryCombe-Richards or BCR algebras. The BCR group is $E(2) \times E(1,1)$, the product of the two-dimensional Euclidean group $E(2)$ with the two-dimensional Poincaré group $E(1,1)$.

If we use light-cone coordinates where $x^{\mu}=$ $\left(x^{-}, x^{+}, x^{i}\right)$, define $\epsilon_{+-12}=+1$, and take the Maxwell field to be zero except for $F^{+-}=1$, then $\mathbf{G}=\mathbf{M}_{+-}$, $\mathbf{G}^{\star}=\mathbf{M}_{12}$, and the algebras associated with the two factors are generated by

$$
E(2):\left\{\mathbf{P}_{1}, \mathbf{P}_{2}, \mathbf{G}^{\star}\right\}, \quad E(1,1):\left\{\mathbf{P}_{+}, \mathbf{P}_{-}, \mathbf{G}\right\} .
$$

For more details on the BCR group, the reader may consult [31].

According to [32], the BCR algebra has three central extensions, so that

$$
\left[\mathbf{G}, \mathbf{G}^{\star}\right]=\mathbf{a}, \quad\left[\mathbf{P}_{\mu}, \mathbf{P}_{\nu}\right]=\mathbf{Z}_{\text {elec }} F_{\mu \nu}+\mathbf{Z}_{\text {mag }} F^{\star}{ }_{\mu \nu} .
$$

There also are four other noncentral charges $\mathbf{Z}_{\mu \nu}$ (in the complement of $\mathbf{Z}_{\mu \nu}^{\text {elec }}=\mathbf{Z}_{\text {elec }} F_{\mu \nu}$ and $\mathbf{Z}_{\mu \nu}^{\mathrm{mag}}=$ $\left.\mathbf{Z}_{\text {mag }} \mathbf{F}^{\star}{ }_{\mu \nu}\right)$ :

$$
\left[\mathbf{P}_{\mu}, \mathbf{P}_{\nu}\right]=\mathbf{Z}_{\text {elec }} F_{\mu \nu}+\mathbf{Z}_{\text {mag }} F_{\mu \nu}^{\star}+\mathbf{Z}_{\mu \nu} .
$$

In total there are six extensions which we can decompose in representations of $\mathbf{G}$ and $\mathbf{G}^{\star}$. The difference with respect to the Maxwell case to be treated later is the presence of two central charges. The central charges are present because the Lorentz group has been reduced to the Abelian subgroup $G_{2}$ generated by $\mathbf{G}$ and $\mathbf{G}^{\star}$. (Note that this twodimensional Abelian group $G_{2}$ is not to be confused with the 14-dimensional non-Abelian simple Lie group of the Cartan/Dynkin classification.)

In $[32,33]$ arguments are given to the effect that if the rotation in $\mathbf{G}, \mathbf{G}^{\star}$ is to be a compact generator then $\mathbf{a}$ should vanish. That leaves $\mathbf{Z}_{\text {elec }}$ and $\mathbf{Z}_{\text {mag }}$, which, as the notation suggests, may be identified with the electric and magnetic charge, respectively. In what follows we shall refer to the eight-dimensional doubly extended kinematic algebra generated by $\left\{\mathbf{P}_{\mu}, \mathbf{G}, \mathbf{G}^{\star}, \mathbf{Z}_{\text {elec }}, \mathbf{Z}_{\text {mag }}\right\}$ as the EBCR algebra.
The EBCR algebra is a direct sum of two subalgebras, each of which has a nontrivial quadratic Casimir. In the case where the only nonvanishing component of $F^{\mu \nu}$ is given by $F^{+-}=1$, the generators of the two subalgebras are $\left\{\mathbf{G}^{\star}, \mathbf{P}_{1}, \mathbf{P}_{2}, \mathbf{Z}_{\text {mag }}\right\}$ and $\left\{\mathbf{G}, \mathbf{P}_{+}, \mathbf{P}_{-}, \mathbf{Z}_{\text {elec }}\right\}$. The two Casimirs are

$$
\begin{aligned}
& \mathbb{C}_{\text {mag }}=\frac{1}{2} \mathbf{P}_{i}^{2}+\mathbf{Z}_{\text {mag }} \mathbf{M}_{12}, \\
& \mathbb{C}_{\text {elec }}=\mathbf{P}_{+} \mathbf{P}_{-}-\mathbf{Z}_{\text {elec }} \mathbf{M}_{+-} .
\end{aligned}
$$

By contrast, the Maxwell algebra is a 16-dimensional extension of the Poincare algebra with the nonvanishing brackets

$$
\begin{gathered}
{\left[\mathbf{M}_{\alpha \beta}, \mathbf{P}_{\gamma}\right]=\eta_{\alpha \gamma} \mathbf{P}_{\beta}-\eta_{\beta \gamma} \mathbf{P}_{\alpha}} \\
{\left[\mathbf{M}_{\alpha \beta}, \mathbf{M}_{\gamma \delta}\right]=} \\
\eta_{\alpha \gamma} \mathbf{M}_{\beta \delta}-\eta_{\alpha \delta} \mathbf{M}_{\beta \gamma}+\eta_{\beta \delta} \mathbf{M}_{\alpha \gamma} \\
-\eta_{\beta \gamma} \mathbf{M}_{\alpha \delta}
\end{gathered}
$$

$\left[\mathbf{M}_{\alpha \beta}, \mathbf{Z}_{\gamma \delta}\right]=\eta_{\alpha \gamma} \mathbf{z}_{\beta \delta}-\eta_{\alpha \delta} \mathbf{z}_{\beta \gamma}+\eta_{\beta \delta} \mathbf{z}_{\alpha \gamma}-\eta_{\beta \gamma} \mathbf{Z}_{\alpha \delta}$,

$$
\left[\mathbf{P}_{\alpha}, \mathbf{P}_{\beta}\right]=\mathbf{Z}_{\alpha \beta},
$$

where $\mathbf{Z}_{\alpha \beta}=-\mathbf{Z}_{\beta \alpha}$. There are two generic Casimirs (which exist for the Maxwell algebra in any dimension) [28],

$$
\mathbb{C}_{1}=\frac{1}{2}\left(\mathbf{P}^{\mu} \mathbf{P}_{\mu}+\mathbf{M}_{\mu \nu} \mathbf{Z}^{\mu \nu}\right), \quad \mathbb{C}_{2}=\frac{1}{2} \mathbf{Z}_{\mu \nu} \mathbf{Z}^{\mu \nu},
$$

and a third quadratic Casimir that exists only in the special case of four dimensions:

$$
\mathbb{C}_{3}=\frac{1}{2} \mathbf{Z}_{\mu \nu} \mathbf{Z}^{\star \mu \nu}
$$

The Casimirs are, of course, elements of the universal enveloping algebra of the Maxwell Lie algebra.

The six additional generators $\mathbf{Z}_{\mu \nu}$ are on the same footing as the Poincare generators $\mathbf{P}_{\mu}$ and $\mathbf{M}_{\mu \nu}$, in that they are dynamical; depending upon the equations of motion of the theory under consideration, the associated momenta may vary with time. The $\mathbf{Z}_{\mu \nu}$ are associated with the Maxwell 2-form. In specific solutions of any equations of motion, there may occur spontaneous symmetry breaking in which the associated momenta $\bar{Z}_{\mu \nu}$ take constant values, $\bar{Z}_{\mu \nu}=-e F_{\mu \nu}{ }^{1}$ The relevant algebra will then reduce to the EBCR algebra that leaves invariant the background field $F_{\mu \nu}$.

A set of left-invariant 1-forms are (we omit those for the Lorentz subalgebra)

$$
P_{L}^{\mu}=d x^{\mu}
$$

\footnotetext{
${ }^{1}$ More precisely, in a Hamiltonian treatment the $\bar{Z}_{\mu \nu}$ are the moment maps. See Sec. VII for further details.
} 


$$
Z_{L}^{\mu \nu}=d \theta^{\mu \nu}-\frac{1}{2}\left(x^{\mu} d x^{\nu}-x^{\mu} d x^{\mu}\right),
$$

with generators of right actions being given by

$$
\begin{gathered}
P_{\mu}^{L}=\frac{\partial}{\partial x^{\mu}}-\frac{1}{2} x^{\nu} \frac{\partial}{\partial \theta^{\mu \nu}}, \\
Z_{\mu \nu}^{L}=\frac{\partial}{\partial \theta^{\mu \nu}} .
\end{gathered}
$$

They satisfy $\left[P_{\mu}^{L}, P_{\nu}^{L}\right]=Z_{\mu \nu}^{L}$.

The 1-forms (2.15) are invariant under

$$
\delta x^{\mu}=\epsilon^{\mu}, \quad \delta \theta^{\mu \nu}=\epsilon^{\mu \nu}+\frac{1}{2}\left(\epsilon^{\mu} x^{\nu}-\epsilon^{\nu} x^{\mu}\right),
$$

which are generated by the vector fields

$$
P_{\mu}^{R}=\frac{\partial}{\partial x^{\mu}}+\frac{1}{2} x^{\nu} \frac{\partial}{\partial \theta^{\mu \nu}}, \quad Z_{\mu \nu}^{R}=\frac{\partial}{\partial \theta^{\mu \nu}} .
$$

The ten-dimensional subalgebra, which is obtained by taking the quotient with respect to the Lorentz subalgebra, is spanned by $\mathbf{P}^{\mu}$ and $\mathbf{Z}^{\mu \nu}$, and closes on the generalized Heisenberg algebra. The associated coset is thus also a group manifold, sometimes called a superspace, and has as coordinates $x^{\mu}$ and $\theta^{\mu \nu}$. This ten-dimensional superspace, which is fibered over Minkowski spacetime with flat six-dimensional fibers, carries a natural Lorentz-invariant metric:

$$
d s_{10}^{2}=\eta_{\mu \nu} P_{L}^{\mu} P_{L}^{\nu}+\frac{1}{2} \eta^{\mu \rho} \eta^{\nu \sigma} Z_{L}^{\mu \nu} Z_{L}^{\rho \sigma} .
$$

\section{A. Quantization}

The obvious approach to quantization is to consider wave functions $\Psi\left(x^{\mu}, \theta^{\mu \nu}\right)$ depending upon both $x^{\mu}$ and $\theta^{\mu \nu}$. A generalized Klein-Gordan or Dirac equation may readily be written in the usual way using the differential operators

$$
P_{\mu}, \quad Z_{\mu \nu} .
$$

The equations can be solved using Fourier transforms, and the solutions used to construct one-particle Hilbert spaces. The Maxwell group acts on these wave functions by pullback, and in this way one obtains a projective representation of the Maxwell group. For details of the procedure, including the calculation of the relevant cocycles, the reader is referred to Schrader's paper [28].

\section{B. Deformations and contractions}

In general dimensions, the Maxwell algebra admits a unique deformation parameter $k$. For $k>0$ we have $\mathfrak{S D}(D-1,2) \oplus \mathfrak{S D}(D-1,1)=\left(\mathcal{M}_{\mu \nu}, \mathcal{P}_{\mu} ; \mathcal{J}_{\mu \nu}\right)$. If instead $k<0$, we have $\mathfrak{S} \mathfrak{D}(D, 1) \oplus \mathfrak{S} \mathfrak{D}(D-1,1)$, where $D=$ $n+2$ is the dimension of spacetime [25,34]. Conversely, it may be regarded as a Wigner-İnönü contraction [25] such that

$$
M_{\mu \nu}=\mathcal{M}_{\mu \nu} \pm \mathcal{J}_{\mu \nu}
$$

$$
P_{\mu}=\lim _{k \rightarrow \infty} \frac{\mathcal{P}_{\mu}}{|k|},
$$

$$
Z_{\mu \nu}=\lim _{k \rightarrow \infty}-\frac{\mathcal{M}_{\mu \nu}}{k^{2}},
$$

where $k$ has the dimensions of length and the sign choice is made depending on whether we consider the anti-de Sitter or de Sitter part.

\section{THE ISIM ALGEBRA}

We may consider a generalization of the discussion of the Maxwell algebra of [35], where the starting point is taken to be the ISim algebra rather than the Poincaré algebra. The ISim generators are

$$
\mathbf{P}_{\mu}, \quad \mathbf{M}_{+i}, \quad \mathbf{M}_{+-}, \quad \mathbf{M}_{i j} .
$$

The ISim algebra, with the conventions we are using, is given in [18].

We define left-invariant 1 -forms $\lambda$ as in (A1), but now, for convenience, we denote them by $\lambda^{a}=$ $\left(P^{\mu}, M^{+i}, M^{+-}, M^{i j}\right)$, and so

$$
g^{-1} d g=P^{\mu} \mathbf{P}_{\mu}+M^{+i} \mathbf{M}_{+i}+\frac{1}{2} M^{i j} \mathbf{M}_{i j}+M^{+-} \mathbf{M}_{+-} .
$$

In terms of these, the $\operatorname{ISim}(n)$ algebra is given by

$$
\begin{aligned}
d P^{+} & =M^{+i} \wedge P^{i}+M^{+-} \wedge P^{+}, \quad d P^{-}=-M^{+-} \wedge P^{-} \\
d P^{i} & =M^{i j} \wedge P^{j}-M^{+i} \wedge P^{-}, \\
d M^{+i} & =M^{i j} \wedge M^{+j}+M^{+-} \wedge M^{+i}, \quad d M^{+-}=0 \\
d M^{i j} & =M^{i k} \wedge M^{k j}
\end{aligned}
$$

The Lie brackets may be immediately read off from the conventions presented in Appendix A.

\section{THE MAXWELL-SIM ALGEBRA}

The Maxwell-Sim algebra can be constructed in complete analogy to the Maxwell algebra discussed previously. One way to describe this it that we start with the $\mathbf{P}_{\mu}$ generators alone, obtain the central extension in which $\left[\mathbf{P}_{\mu}, \mathbf{P}_{\nu}\right]=\mathbf{Z}_{\mu \nu}$, and then append the $\operatorname{Sim}(n)$ generators $\left(\mathbf{M}_{+i}, \mathbf{M}_{+-}, \mathbf{M}_{i j}\right)$ to form the Maxwell-Sim $(n)$ algebra. At the level of the left-invariant 1-forms, this means that we augment the $\operatorname{ISim}(n)$ relations (3.3) by

$$
\begin{aligned}
d Z^{+i}= & -P^{+} \wedge P^{i}+M^{+-} \wedge Z^{+i}+M^{i j} \wedge Z^{+j} \\
& +M^{+i} \wedge Z_{-+}-M^{+j} \wedge Z^{i j}, \\
d Z^{-i}= & -P^{-} \wedge P^{i}-M^{+-} \wedge Z^{-i}+M^{i j} \wedge M^{+j}, \\
d Z^{-+}= & -P^{-} \wedge P^{+}+M^{+i} \wedge Z^{-i}, \\
d Z^{i j}= & -P^{i} \wedge P^{j}-M^{+i} \wedge Z^{-j}+M^{+j} \wedge Z^{-i} .
\end{aligned}
$$




\section{DEFORMATIONS OF MAXWELL-Sim $(n)$}

We follow the method for finding the general nontrivial deformation of an algebra that is described in [18] (see [36] for further details). This entails first finding the second cohomology class $H^{2}(\mathfrak{g}, \mathfrak{g})$, which determines the nontrivial deformations at the linear level. If $H^{3}(\mathfrak{g}, \mathfrak{g})$ is trivial, then there must exist, possibly after making (trivial) redefinitions, an extension of the linearized deformations that is valid to all orders. (This is checked by verifying that the deformed algebra satisfies the Jacobi identities.) If, on the other hand, $H^{3}(\mathfrak{g}, \mathfrak{g})$ is nontrivial, then the extension beyond the linearized level may not be possible.

For the generic case of the Maxwell-Sim( $n)$ algebra, we find that there are two distinct 1-parameter nontrivial deformations. We denote these by the $b$ deformation and the $c$ deformation, where $b$ and $c$ are the respective constants parametrizing the two deformations. ${ }^{2}$

\section{A. The $b$ deformation}

In the $b$ deformation, the Maxwell-Sim( $n)$ algebra defined by (3.3) and (4.1) is modified by the following additions to $d P^{\mu}$ and $d Z^{\mu \nu}$ :

$$
\begin{aligned}
d P^{\mu} & =b M^{+-} \wedge P^{\mu}+\cdots, \\
d Z^{\mu \nu} & =2 b M^{+-} \wedge Z^{\mu \nu}+\cdots,
\end{aligned}
$$

where the "..." terms represent the usual right-hand sides of the undeformed Maxwell-Sim $(n)$ algebra. The $\operatorname{Sim}(n)$ relations in (3.3) are unmodified.

\section{B. The $c$ deformation}

In the $c$ deformation, the Maxwell-Sim( $n)$ algebra defined by (3.3) and (4.1) is modified by the following additions to $d P^{\mu}$ and $d Z^{\mu \nu}$ :

$$
\begin{aligned}
d P^{\mu} & =c P_{\nu} \wedge Z^{\mu \nu}+\cdots, \\
d Z^{\mu \nu} & =-c Z_{\rho}^{\mu} \wedge Z^{\rho \nu}+\cdots,
\end{aligned}
$$

where again the "..." terms represent the usual right-hand sides of the undeformed Maxwell-Sim( $n)$ algebra. The $\operatorname{Sim}(n)$ relations in (3.3) are again unmodified.

It is not possible to turn on the $b$ and $c$ deformations simultaneously. (This agrees with the fact that there are no de Sitter or anti-de Sitter deformations of the ISim algebra [18].)

In the special case of Maxwell-Sim(2), we find that there is an additional nontrivial deformation characterized by a parameter $a$, which can be turned on simultaneously with the $b$ deformation. Thus in place of the $b$ deformation given by (5.1), for Maxwell-Sim(2) we may have

\footnotetext{
${ }^{2}$ We have performed some of the calculations with differential forms with the aid of the EDC MATHEMATICA package [37].
}

$$
\begin{aligned}
d P^{\mu} & =a M^{12} \wedge P^{\mu}+b M^{+-} \wedge P^{\mu}+\cdots, \\
d Z^{\mu \nu} & =2 a M^{12} \wedge Z^{\mu \nu}+2 b M^{+-} \wedge Z^{\mu \nu}+\cdots
\end{aligned}
$$

A calculation of the cohomology group $H^{3}(\mathfrak{g}, \mathfrak{g})$ for Maxwell-Sim(2) shows that it is nontrivial, and of dimension three.

Note that the deformation parametrized by $c$ in (5.2) is analogous to the $k$ deformation of the Maxwell algebra found in [25], now restricted to the 14 generators of Maxwell-Sim(2), which gave $S O(3,2) \times S O(3,1)$ or $S O(4,1) \times S O(3,1)$ depending on the sign of $k$.

The deformations associated with the parameters $a$ and $b$ are the Maxwellian extensions of the $a$ and $b$ deformations of the ISim(2) algebra obtained in [18].

\section{LAGRANGIANS}

\section{A. The Maxwell case}

A particle model can be derived geometrically by the techniques of nonlinear realizations [26]. Let us first consider the coset (Maxwell)/(Lorentz). In order to construct a Lorentz-invariant Lagrangian from the Maurer-Cartan forms (2.15), one possibility is to introduce new dynamical variables $f_{\mu \nu}$ that transform covariantly under the Maxwell group [38]. A Lagrangian containing only first derivatives with respect to proper time may be taken to be

$$
L=-m \sqrt{-\dot{x}^{2}}+\frac{1}{2} f_{\mu \nu}\left(\dot{\theta}^{\mu \nu}-\frac{1}{2}\left(x^{\mu} \dot{x}^{\nu}-x^{\nu} \dot{x}^{\mu}\right)\right)
$$

The equations of motion in the proper-time gauge are

$$
\begin{gathered}
\dot{f}_{\mu \nu}=0, \\
\dot{\theta}^{\mu \nu}=\frac{1}{2}\left(x^{\mu} \dot{x}^{\nu}-x^{\nu} \dot{x}^{\mu}\right), \\
m \ddot{x}_{\mu}=f_{\mu \nu} \dot{x}^{\nu} .
\end{gathered}
$$

Integration of (6.2) gives $f_{\mu \nu}=f_{\mu \nu}^{0}$, where $f_{\mu \nu}^{0}$ is a constant tensor. This solution spontaneously breaks the Lorentz symmetry algebra down to a subalgebra of the Maxwell algebra (namely the EBCR algebra discussed earlier). Substituting this solution into Eq. (6.4) gives the motion of a particle in a constant electromagnetic field.

The method of nonlinear realizations does not provide a unique invariant Lagrangian. In fact since we know how to construct Lorentz scalars, we can construct a Lagrangian without the introduction of the new dynamical variables $f_{\mu \nu}$ as

$$
L=m \dot{x}^{2}+\frac{\alpha}{2}\left(\dot{\theta}^{\mu \nu}-\frac{1}{2}\left(x^{\mu} \dot{x}^{\nu}-x^{\nu} \dot{x}^{\mu}\right)\right)^{2},
$$

where $\alpha$ is a constant. The quantities $\dot{\theta}^{\mu \nu}-\frac{1}{2} \times$ $\left(x^{\mu} \dot{x}^{\nu}-x^{\nu} \dot{x}^{\mu}\right)$ are constants of motion. Choosing $\dot{\theta}^{\mu \nu}-$ $\frac{1}{2}\left(x^{\mu} \dot{x}^{\nu}-x^{\nu} \dot{x}^{\mu}\right)=-\alpha^{-1} f_{\mu \nu}^{0}$, we recover the same equation of motion of a particle moving in a constant electromagnetic field that we obtained above. 
Another way to construct the Lagrangian is to consider the coset (Maxwell)/(rotations). This coset is useful for the construction of massive particle Lagrangians when the tensor calculus is not known. For example, in the case of ISim, we may consider the coset (ISim)/(rotations), rather than (ISim)/(Sim), because we do not know a priori what is the length element; in order words, we do not have an obvious tensorial calculus. A more striking example is the case of the deformed ISim algebra DISim [18]. We obtain left-invariant 1 -forms, by first defining

$$
g=g_{0} U,
$$

where

$$
g_{0}=e^{x^{\mu} P_{\mu}} e^{(1 / 2) \theta^{\mu \nu} Z_{\mu \nu}}, \quad U=e^{w^{i} M_{0 i}},
$$

and $w^{i}, i=1,2,3$, are the Goldstone bosons associated with the broken boost symmetry generators. The leftinvariant 1 -forms $\lambda$ may then be read off from

$$
\begin{aligned}
g^{-1} d g & =U^{-1} g_{0}^{-1} d g_{0} U+U^{-1} d U \\
& =\lambda_{P}^{\mu} P_{\mu}+\lambda_{Z}^{\mu \nu} Z_{\mu \nu}+\lambda_{M}^{i} M_{0 i}+\lambda_{R}^{i j} M_{i j} .
\end{aligned}
$$

Defining

$$
g_{0}^{-1} d g_{0}=\bar{\lambda}_{P}^{\mu} P_{\mu}+\bar{\lambda}_{Z}^{\mu \nu} Z_{\mu \nu}
$$

we have

$$
\bar{\lambda}_{P}^{\mu}=d x^{\mu}, \quad \bar{\lambda}_{Z}^{\mu \nu}=d \theta^{\mu \nu}-\frac{1}{2}\left(x^{\mu} d x^{\nu}-x^{\nu} d x^{\mu}\right) .
$$

The Lorentz transformations generated by $U$ may be used to define $\Lambda_{\mu}^{\nu}\left(w^{i}\right)$ :

$$
U^{-1} P_{\mu} U=\Lambda_{\mu}^{\nu}\left(w^{i}\right) P_{\nu} .
$$

The left-invariant 1 -forms $\lambda_{P}^{\mu}, \lambda_{R}^{i j}$, and $\lambda_{Z}^{\mu \nu}$ are then given by

$$
\begin{aligned}
\lambda_{P}^{\mu} & =\Lambda^{\mu}{ }_{\nu} \bar{\lambda}_{P}^{\nu}=\Lambda^{\mu}{ }_{\nu} d x^{\nu}, \\
\lambda_{Z}^{\mu \nu} & =\Lambda^{\mu}{ }_{\rho} \Lambda^{\nu}{ }_{\sigma} \bar{\lambda}_{Z}^{\rho \sigma} \\
& =\Lambda^{\mu}{ }_{\rho} \Lambda^{\nu}{ }_{\sigma}\left[d \theta^{\rho \sigma}-\frac{1}{2}\left(x^{\rho} d x^{\sigma}-x^{\sigma} d x^{\rho}\right)\right] .
\end{aligned}
$$

The 1-forms $\lambda_{M}^{i}$ are given by

$$
\begin{aligned}
\lambda_{M}^{i} & =d w^{i}+d w^{j}\left(\delta_{j}^{i}-w^{i} w^{j}\right)\left(\frac{\sinh w}{w}-1\right), \\
\lambda_{R}^{i j} & =\left(\frac{d w^{i} w^{j}-d w^{j} w^{i}}{w^{2}}\right)(\cosh w-1) .
\end{aligned}
$$

A particle Lagrangian that is invariant under $S O(3)$ is

$$
L=\left[m \lambda_{P}^{0}+\frac{1}{2} \hat{f}_{\mu \nu}(\tau) \lambda_{Z}^{\mu \nu}\right]^{*},
$$

where the $*$ indicates that the 1 -forms are pulled back onto the worldline: $\left[d x^{\mu}\right]^{*} \equiv \dot{x}^{\mu}(\tau) d \tau$, etc. The coefficient $m$ is the mass of the particle, while $\hat{f}_{\mu \nu}(\tau)$ is a dynamical field that depends upon $\tau$. We see that (6.14) may be written as

$$
L=m \Lambda_{\mu}^{0} \dot{x}^{\mu}+\frac{1}{2} f_{\mu \nu}\left[\dot{\theta}^{\mu \nu}-\frac{1}{2}\left(x^{\mu} \dot{x}^{\nu}-x^{\nu} \dot{x}^{\mu}\right)\right],
$$

where $\Lambda_{\nu}^{\mu}$ is a general Lorentz boost transformation and depends on the nondynamical coordinates $w^{i}$ which are the Goldstone bosons associated to the broken boost. The presence of nondynamical Goldstone bosons in the nonlinear realization approach was first noticed in [9]. We have also introduced the tensor field $f_{\mu \nu}$, which is related to $\hat{f}_{\mu \nu}$ by

$$
f_{\mu \nu}=\Lambda_{\mu}^{\rho}\left(w^{i}\right) \Lambda_{\nu}^{\sigma}\left(w^{i}\right) \hat{f}_{\rho \sigma} .
$$

We now define the particle momentum $p_{\mu}$ in the canonical way: ${ }^{3}$

$$
p_{\mu}=\frac{\partial L}{\partial \dot{x}^{\mu}}=m \Lambda^{0}{ }_{\mu}+\frac{1}{2} f_{\mu \nu} x^{\nu} .
$$

Because $\Lambda^{0}{ }_{\mu}$ is a timelike Lorentz vector, we have

$$
\left(p_{\mu}-\frac{1}{2} f_{\mu \nu} x^{\nu}\right)^{2}=-m^{2} .
$$

Introducing $e$ as a Lagrange multiplier to enforce the massshell condition (6.18), we arrive at the Lagrangian

$$
\begin{aligned}
L= & \left(p_{\mu}-\frac{1}{2} f_{\mu \nu} x^{\nu}\right) \dot{x}^{\mu}+\frac{1}{2} f_{\mu \nu}\left[\dot{\theta}^{\mu \nu}-\frac{1}{2}\left(x^{\mu} \dot{x}^{\nu}-x^{\nu} \dot{x}^{\mu}\right)\right] \\
& -\frac{1}{2} e\left[\left(p_{\mu}-\frac{1}{2} f_{\mu \nu} x^{\nu}\right)^{2}+m^{2}\right] .
\end{aligned}
$$

Varying with respect to $p_{\mu}$ gives

$$
\dot{x}^{\mu}=e\left(p^{\mu}-\frac{1}{2} f^{\mu}{ }_{\nu} x^{\nu}\right) .
$$

Substituting for $p_{\mu}$ in (6.19), and then varying with respect to $e$ to obtain

$$
e=-\frac{\sqrt{-\dot{x}^{2}}}{m}
$$

we finally arrive at the Lagrangian (6.1). In Sec. VII we shall see how the nonlinear realization method and coadjoint orbit technique gives the same results.

\section{B. The Maxwell-Sim Lagrangian}

We start with the coset (Maxwell-Sim)/SO(2), and then construct the left-invariant 1-forms from the coset representative

$$
g=g_{0} U,
$$

with

$$
g_{0}=e^{x^{\mu} P_{\mu}} e^{(1 / 2) \theta^{\mu \nu} Z_{\mu \nu}}, \quad U=e^{w^{i} M_{+i}} e^{w M_{+-}} .
$$

Following the same steps as in the Maxwell case, we have

$$
g^{-1} d g=\lambda_{P}^{\mu} P_{\mu}+\lambda_{Z}^{\mu \nu} Z_{\mu \nu}+\lambda_{M}^{i} M_{+i}+\lambda_{M_{+-}} M_{+-} .
$$

\footnotetext{
${ }^{3}$ In the introduction, we used $\pi$ to denote the canonical momentum, but from now on we shall use $p_{\mu}$ for this purpose.
} 
The left-invariant 1-forms $\lambda_{P}^{\mu}$ and $\lambda_{Z}^{\mu \nu}$ are then given by

$$
\begin{aligned}
\lambda_{P}^{\mu} & =\Lambda_{\nu}^{\mu}{ }_{\nu} \bar{\lambda}_{P}^{\nu}=\Lambda_{\nu}^{\mu} d x^{\nu}, \\
\lambda_{Z}^{\mu \nu} & =\Lambda^{\mu}{ }_{\rho} \Lambda^{\nu}{ }_{\sigma} \bar{\lambda}_{Z}^{\rho \sigma} \\
& =\Lambda^{\mu}{ }_{\rho} \Lambda^{\nu}{ }_{\sigma}\left[d \theta^{\rho \sigma}-\frac{1}{2}\left(x^{\rho} d x^{\sigma}-x^{\sigma} d x^{\rho}\right)\right],
\end{aligned}
$$

where

$$
\bar{\lambda}_{P}^{\mu}=d x^{\mu}, \quad \bar{\lambda}_{Z}^{\mu \nu}=d \theta^{\mu \nu}-\frac{1}{2}\left(x^{\mu} d x^{\nu}-x^{\nu} d x^{\mu}\right) .
$$

The Lorentz transformation $\Lambda_{\nu}^{\mu}\left(w^{i}, w\right)$ is given by

$$
\Lambda_{\nu}^{\mu}=\left(\begin{array}{ccc}
e^{-w} & 0 & 0 \\
-\frac{1}{2} e^{w} w^{k} w^{k} & e^{w} & e^{w} w^{i} \\
-w^{j} & 0 & 1
\end{array}\right)
$$

where we order the spacetime coordinates in the sequence $x^{\mu}=\left(x^{-}, x^{+}, x^{i}\right), i=1,2$.

The 1-forms $\lambda_{M}^{i}$ and $\lambda_{N}$ are given by

$$
\lambda_{M}^{i}=e^{w} d w^{i}, \quad \lambda_{N}=d w .
$$

A particle Lagrangian that is invariant under $S O(2)$ (generated by $J=M_{12}$ ) is given by

$$
L=\left[\alpha \lambda_{P}^{+}-\beta \lambda_{P}^{-}+\frac{1}{2} \hat{f}_{\mu \nu}(\tau) \lambda_{Z}^{\mu \nu}\right]^{*} .
$$

As in the Maxwell case the coefficients $\alpha$ and $\beta$ are constants, while $\hat{f}_{\mu \nu}(\tau)$ is a dynamical field that depends upon $\tau$. We see that (6.29) may be written as

$$
\begin{aligned}
L= & \alpha \Lambda^{+}{ }_{\mu} \dot{x}^{\mu}-\beta \Lambda_{\mu}^{-} \dot{x}^{\mu} \\
& +\frac{1}{2} f_{\mu \nu}\left[\dot{\theta}^{\mu \nu}-\frac{1}{2}\left(x^{\mu} \dot{x}^{\nu}-x^{\nu} \dot{x}^{\mu}\right)\right],
\end{aligned}
$$

where

$$
f_{\mu \nu}=\Lambda_{\mu}^{\rho}\left(w, w^{i}\right) \Lambda^{\sigma}{ }_{\nu}\left(w, w^{i}\right) \hat{f}_{\rho \sigma} .
$$

The particle momentum $p_{\mu}$ is given by

$$
p_{\mu}=\frac{\partial L}{\partial \dot{x}^{\mu}}=\alpha \Lambda_{\mu}^{+}{ }_{\mu}-\beta \Lambda^{-}{ }_{\mu}+\frac{1}{2} f_{\mu \nu} x^{\nu} .
$$

Noting that $\Lambda^{+} \mu \Lambda^{+\mu}=\Lambda^{-}{ }_{\mu} \Lambda^{-\mu}=0$ and $\Lambda^{+}{ }_{\mu} \Lambda^{-\mu}=$ 1 , we see that

$$
\left(p_{\mu}-\frac{1}{2} f_{\mu \nu} x^{\nu}\right)^{2}=-m^{2},
$$

where we have defined the mass parameter as

$$
m=\sqrt{2 \alpha \beta} .
$$

Introducing $e$ as a Lagrange multiplier to enforce the massshell condition (6.33), we arrive at the Lagrangian

$$
\begin{aligned}
L= & \left(p_{\mu}-\frac{1}{2} f_{\mu \nu} x^{\nu}\right) \dot{x}^{\mu}+\frac{1}{2} f_{\mu \nu}\left[\dot{\theta}^{\mu \nu}-\frac{1}{2}\left(x^{\mu} \dot{x}^{\nu}-x^{\nu} \dot{x}^{\mu}\right)\right] \\
& -\frac{1}{2} e\left[\left(p_{\mu}-\frac{1}{2} f_{\mu \nu} x^{\nu}\right)^{2}+m^{2}\right] .
\end{aligned}
$$

Varying with respect to $p_{\mu}$ gives

$$
\dot{x}^{\mu}=e\left(p^{\mu}-\frac{1}{2} f^{\mu}{ }_{\nu} x^{\nu}\right) .
$$

Substituting for $p_{\mu}$ in (6.35), and then varying with respect to $e$, we get the Lagrangian (6.1). Thus the undeformed Maxwell-Sim algebra gives the same particle Lagrangian as the Maxwell algebra based on the full Poincaré group.

\section{The Maxwell-DISim ${ }_{b}$ Lagrangian}

The left-invariant 1-forms $\lambda_{P}^{\mu}$ of the $\mathrm{DISim}_{b}$ algebra are given by

$$
\lambda_{P}^{\mu}=\tilde{\Lambda}_{\nu}^{\mu} d x^{\nu},
$$

where the matrix $\tilde{\Lambda}$ is

$$
\tilde{\Lambda}^{\mu}{ }_{\nu}=\left(\begin{array}{ccc}
e^{-w(1-b)} & 0 & 0 \\
-\frac{1}{2} e^{w(1+b)} w^{k} w^{k} & e^{w(1+b)} & e^{w(1+b)} w^{i} \\
-w^{j} & 0 & 1
\end{array}\right) .
$$

The 1-forms $\lambda_{M}^{i}$ and $\lambda_{N}$ are given by

$$
\lambda_{M}^{i}=e^{w} d w^{i}, \quad \lambda_{N}=d w .
$$

We wish to construct a particle Lagrangian that is invariant under $S O(2)$ (generated by $J=M_{12}$ ). Thus we begin by writing

$$
L=\left[\alpha \lambda_{P}^{+}-\beta \lambda_{P}^{-}\right]^{*},
$$

where as before the $*$ indicates that the 1 -forms are pulled back onto the worldline. The coefficients $\alpha$ and $\beta$ are constants. We see that (6.40) may be written as

$$
L=\alpha \tilde{\Lambda}_{\mu}^{+} \dot{x}^{\mu}-\beta \tilde{\Lambda}^{-}{ }_{\mu} \dot{x}^{\mu},
$$

where $\tilde{\Lambda}^{\mu}{ }_{\nu}$ depends on the nondynamical coordinates $w$ and $w^{i}$, and is given by (6.38).

We now define the particle momentum $p_{\mu}$ in the canonical way:

$$
p_{\mu}=\frac{\partial L}{\partial \dot{x}^{\mu}}=\alpha \tilde{\Lambda}_{\mu}^{+}-\beta \tilde{\Lambda}_{\mu}^{-} .
$$

Noting that $\tilde{\Lambda}^{+} \mu \tilde{\Lambda}^{+\mu}=\tilde{\Lambda}^{-}{ }_{\mu} \tilde{\Lambda}^{-\mu}=0$ and

$$
\tilde{\Lambda}^{+}{ }_{\mu} \tilde{\Lambda}^{-\mu}=e^{2 w b},
$$

we have the constraint

$$
p^{2}=-2 \alpha \beta\left(\frac{p_{+}}{\alpha}\right)^{((2 b) /(1+b))}=-2 \alpha \beta\left(\frac{n^{\mu} p_{\mu}}{\alpha}\right)^{((2 b) /(1+b))} \text {. }
$$

With $\alpha=-m(1-b)$ and $\beta=-\frac{1}{2} m(1+b)$ we obtain Eq. (18) of [18]:

$$
p^{2}+m^{2}\left(1-b^{2}\right)\left(-\frac{n^{\nu} p_{\nu}}{m(1-b)}\right)^{2 b /(1+b)}=0 .
$$


Introducing $e$ as a Lagrange multiplier to enforce the massshell condition (6.45), we arrive at the Lagrangian

$$
\begin{aligned}
L= & p_{\mu} \dot{x}^{\mu}-\frac{1}{2} e\left[p^{2}+m^{2}\left(1-b^{2}\right)\right. \\
& \left.\times\left(-\frac{n^{\nu} p_{\nu}}{m(1-b)}\right)^{2 b /(1+b)}\right] .
\end{aligned}
$$

Varying with respect to $p_{\mu}$ gives

$$
\dot{x}^{\mu}=e\left[p^{\mu}-b m\left(-\frac{n^{\nu} p_{\nu}}{m(1-b)}\right)^{((b-1) /(b+1))} n^{\mu}\right] .
$$

If we solve for $p_{\mu}$ and substitute into (6.46), we obtain

$$
\begin{aligned}
L= & \frac{1}{2} \frac{\dot{x}^{2}}{e}-\frac{1}{2} m^{2}\left(1-b^{2}\right) \\
& \times\left(-\frac{n^{\nu} \dot{x}_{\nu}}{m(1-b)}\right)^{2 b /(1+b)} e^{(1-b) /(1+b)} .
\end{aligned}
$$

Varying this with respect to $e$ we get

$$
e=\frac{1}{m(1-b)}\left(-\dot{x}^{2}\right)^{((1+b) / 2)}\left(-n^{\nu} \dot{x}_{\nu}\right)^{-b},
$$

from which we obtain the Finslerian Lagrangian of [18]

$$
L=-m\left(-\eta_{\mu \nu} \dot{x}^{\mu} \dot{x}^{\nu}\right)^{(1-b) / 2}\left(-n_{\rho} \dot{x}^{\rho}\right)^{b} .
$$

For the Maxwell-DISim ${ }_{b}$ case, following the same steps as for the Maxwell-Sim case, we get

$$
\begin{aligned}
L= & -m\left(-\eta_{\mu \nu} \dot{x}^{\mu} \dot{x}^{\nu}\right)^{(1-b) / 2}\left(-n_{\rho} \dot{x}^{\rho}\right)^{b} \\
& +\frac{1}{2} f_{\mu \nu}\left(\dot{\theta}^{\mu \nu}-\frac{1}{2}\left(x^{\mu} \dot{x}^{\nu}-x^{\nu} \dot{x}^{\mu}\right)\right) .
\end{aligned}
$$

\section{HAMILTONIAN VIEWPOINT}

\section{A. Kaluza-Klein interlude}

Before dealing with the Maxwell algebra approach, it may be helpful to contrast the six "angles" $\theta_{\mu \nu}$ with the single angle $\theta$ introduced in Kaluza-Klein approaches to motion in a homogeneous electromagnetic field, considered as geodesic motion in the five-dimensional Heisenberg group. The Maurer-Cartan forms are

$$
P^{\mu}=d x^{\mu}, \quad Z=d \theta-\frac{1}{2} F_{\mu \nu} x^{\mu} d x^{\nu},
$$

with the nontrivial algebra

$$
d Z=-\frac{1}{2} F_{\mu \nu} P^{\mu} \wedge P^{\nu},
$$

and metric

$$
d s_{5}^{2}=\eta_{\mu \nu} d x^{\mu} d x^{\nu}+\left(d \theta-\frac{1}{2} F_{\mu \nu} x^{\mu} d x^{\nu}\right)^{2} .
$$

The metric (7.3) is invariant under the left action of the Heisenberg group, and an additional outer action of the Abelian subgroup $G_{2} \subset S O(3,1)$ generated by $\mathbf{G}$ and $\mathbf{G}^{\star}$. We may identify $\mathbf{Z}$ with $\mathbf{Z}_{\text {elec }}$ introduced earlier. If we consider the coset $(\mathrm{EBCR}) /\left(G_{2}, Z_{\text {mag }}\right)$, then the quadratic combination $P^{2}+Z^{2}$ is invariant under the stability group.
The corresponding metric is (7.3) and therefore it is invariant under the whole EBCR group.

A convenient matrix representation of the Heisenberg group is given by

$$
\left(\begin{array}{c}
x^{\prime \mu} \\
\theta^{\prime} \\
1
\end{array}\right)=\left(\begin{array}{ccc}
\delta_{\nu}^{\mu} & 0 & a^{\mu} \\
-\frac{1}{2} F_{\nu \lambda} a^{\lambda} & 1 & \alpha \\
0 & 0 & 1
\end{array}\right)\left(\begin{array}{c}
x^{\nu} \\
\theta \\
1
\end{array}\right) .
$$

The phase or cotangent space $T^{\star}(G) \equiv G \times \mathfrak{g}$ of the Heisenberg algebra has coordinates $\left(x^{\mu}, \theta, p_{\mu}, p_{\theta}\right)$. $\left(\bar{P}_{\mu}, \bar{M}_{\mu \nu}, \bar{Z}\right)$ are the corresponding moment maps generating right actions, and are given by

$$
\bar{P}_{\mu}=p_{\mu}-\frac{1}{2} p_{\theta} F_{\mu \nu} x^{\nu}, \quad \bar{Z}=p_{\theta} .
$$

The nonvanishing Poisson brackets of the generators of the right actions of the Heisenberg group are

$$
\left\{\bar{P}_{\mu}, \bar{P}_{\nu}\right\}=-F_{\mu \nu} \bar{Z} .
$$

The geodesic Hamiltonian associated to the metric (7.3) is

$$
H=\frac{1}{2 m} \bar{P}_{\mu} \bar{P}^{\mu}+\frac{1}{2 m} \bar{Z}^{2},
$$

so that

$$
\dot{\bar{Z}}=0, \quad \dot{\bar{P}}_{\mu}=-\frac{1}{m} \bar{Z} F_{\mu \nu} \bar{P}^{\nu} .
$$

[Note that although the $\bar{Z}^{2}$ term in (7.7) is needed for the correspondence with the metric (7.3), it plays no role in the dynamics.] The $x^{\mu}$ equation of motion is

$$
\dot{x}^{\mu}=\frac{1}{m} \bar{P}^{\mu} .
$$

The equation for $\theta$, conjugate to $\bar{Z}_{\mu \nu}$, is

$$
\dot{\theta}=\frac{1}{m} \bar{Z} .
$$

The moment maps that generate left translations are given by

$$
\bar{P}_{\mu}+\bar{Z} F_{\mu \nu} x^{\nu}, \quad \bar{Z} .
$$

These are constant for a Hamiltonian such as (7.7), which depends only on the moment maps that generate right translations.

The mechanical momentum $p_{i}$ and Noether momentum $P_{i}$ in our discussion in the introduction correspond to momentum maps generating right translations and left translations, respectively.

We have obtained the standard Lorentz force equation, and the electric charge corresponds to the conserved momentum $-\bar{Z}$ in the extra dimension. The externally given Maxwell field $F_{\mu \nu}$ is constant throughout, and is nondynamical. Note that we could obtain the same result with a more general Hamiltonian of the form 


$$
H=\frac{1}{2 m} \bar{P}_{\mu} \bar{P}^{\mu}+\beta \bar{Z}^{2},
$$

where $\beta$ is an arbitrary constant.

We may also include a magnetic charge by adding an extra central extension

$$
Z^{\star}=d \theta^{\star}-\frac{1}{2} F^{\star}{ }_{\mu \nu} x^{\mu} d x^{\nu} .
$$

The Hamiltonian

$$
H=\frac{1}{2 m} \bar{P}_{\mu} \bar{P}^{\mu}
$$

will now lead to the constancy of both $\bar{Z}$ and $\bar{Z}^{\star}$ and the equation of motion

$$
\dot{\bar{P}}_{\mu}=-\frac{1}{m}\left(\bar{Z} F_{\mu \nu}+\bar{Z}^{\star} F^{\star}{ }_{\mu \nu}\right) \bar{P}^{\nu}
$$

If we identify $\mathbf{Z}^{\star}$ with $\mathbf{Z}_{\text {mag }}$, then the six-dimensional Heisenberg algebra with two central charges may be identified with the coset $(\mathrm{EBCR}) / G_{2}$. Note that the presence of magnetic and electric charges is due to the presence of central charges in the eight-dimensional EBCR algebra. These central charges are absent in the Maxwell algebra.

\section{B. The Maxwell algebra}

The phase space, or cotangent space, $T^{\star}(G) \equiv G \times \mathfrak{g}$ of the Maxwell algebra has coordinates $\left(x^{\mu}, \theta^{\mu \nu}, p_{\mu}, f_{\mu \nu}\right)$. The left-invariant Maurer-Cartan forms are given by (2.15) and $\left(\bar{P}_{\mu}, \bar{M}_{\mu \nu}, \bar{Z}_{\mu \nu}\right)$ are the corresponding moment maps generating right actions. They are given by

$$
\bar{P}_{\mu}=p_{\mu}-\frac{1}{2} f_{\mu \nu} x^{\nu}, \quad \bar{Z}_{\mu \nu}=f_{\mu \nu} .
$$

The nonvanishing Poisson brackets are

$$
\begin{gathered}
\left\{\bar{M}_{\alpha \beta}, \bar{P}_{\gamma}\right\}=\eta_{\beta \gamma} \bar{P}_{\alpha}-\eta_{\alpha \gamma} \bar{P}_{\beta} \\
\left\{\bar{M}_{\alpha \beta}, \bar{M}_{\gamma \delta}\right\}=\eta_{\beta \gamma} \bar{M}_{\alpha \delta}-\eta_{\beta \delta} \bar{M}_{\alpha \gamma}+\eta_{\alpha \delta} \bar{M}_{\beta \gamma} \\
-\eta_{\alpha \gamma} \bar{M}_{\beta \delta}, \\
\left\{\bar{M}_{\alpha \beta}, \bar{Z}_{\gamma \delta}\right\}=\eta_{\beta \gamma} \bar{Z}_{\alpha \delta}-\eta_{\beta \delta} \bar{Z}_{\alpha \gamma}+\eta_{\alpha \delta} \bar{Z}_{\beta \gamma}-\eta_{\alpha \gamma} \bar{Z}_{\beta \delta}, \\
\left\{\bar{P}_{\alpha}, \bar{P}_{\beta}\right\}=-\bar{Z}_{\alpha \beta} .
\end{gathered}
$$

There are two generic Casimir functions,

$$
C_{1}=\frac{1}{2}\left(\bar{P}^{\mu} \bar{P}_{\mu}+\bar{M}_{\mu \nu} \bar{Z}^{\mu \nu}\right), \quad C_{2}=\frac{1}{2} \bar{Z}_{\mu \nu} \bar{Z}^{\mu \nu} .
$$

For the Hamiltonian, we take

$$
H=\frac{1}{2 m} \eta^{\mu \nu} \bar{P}_{\mu} \bar{P}_{\nu}
$$

Thus the Euler equations imply

$$
\dot{\bar{Z}}_{\mu \nu}=0, \quad \Rightarrow \bar{Z}_{\mu \nu}=-e F_{\mu \nu}=\text { constant },
$$

$$
\dot{\bar{P}}_{\mu}=\frac{1}{m}\left\{\bar{P}_{\mu}, \bar{P}_{\nu}\right\} \bar{P}^{\nu}=-\bar{Z}_{\mu \nu} \bar{P}^{\nu}=e F_{\mu \nu} \bar{P}^{\nu},
$$

and the $x^{\mu}$ equation of motion is

$$
\dot{x}^{\mu}=\frac{1}{m} \bar{P}^{\mu} .
$$

The equation for $\theta^{\mu \nu}$, conjugate to $\bar{Z}_{\mu \nu}$ is

$$
\dot{\theta}^{\mu \nu}=\frac{1}{2}\left(x^{\mu} \dot{x}^{\nu}-x^{\nu} \dot{x}^{\mu}\right) .
$$

Thus we obtain the motion of a particle in a constant electromagnetic field, for which the momentum vector $\bar{P}^{\mu}(\tau)$ undergoes a constant Lorentz transformation

$$
\bar{P}^{\mu}(\tau)=[\exp (e \tau F)]_{\nu}^{\mu} \bar{P}^{\nu}(0) .
$$

By contrast with the Kaluza-Klein approach, which gives the same equations for the $x^{\mu}$ variables with an externally imposed constant Maxwell field $F_{\mu \nu}$, in the Maxwell algebra approach we find that the Maxwell field must be constant as a consequence of the equations of motion. The equations for the six angles $\theta^{\mu \nu}$ are also richer. They may be interpreted geometrically as follows. The curve in spacetime $x^{\mu}=x^{\mu}(\tau)$ has a projection onto each $\mu-\nu$ 2-plane. The curve sweeps out area at a rate

$$
\frac{d A^{\mu \nu}}{d \tau}=\frac{1}{2}\left(x^{\mu} \dot{x}^{\nu}-x^{\nu} \dot{x}^{\mu}\right) .
$$

Thus (7.26) may be rewritten as

$$
\frac{d \theta^{\mu \nu}}{d \tau}=\frac{d A^{\mu \nu}}{d \tau} .
$$

In other words $\theta^{\mu \nu}(\tau)$ is the total area $A^{\mu \nu}(\tau)$ swept out during the motion.

The canonical Lagrangian that reproduces the previous equation of motion is

$$
L=\bar{P}_{\mu} \dot{x}^{\mu}+\frac{1}{2} f_{\mu \nu}\left[\dot{\theta}^{\mu \nu}-\frac{1}{2}\left(x^{\mu} \dot{x}^{\nu}-x^{\nu} \dot{x}^{\mu}\right)\right]-\frac{1}{2} e \bar{P}^{2},
$$

which, apart from a constant piece, is obtained from the diffeomorphism-invariant Lagrangian (6.19) by choosing the proper-time gauge $e=m$.

One may choose different Hamiltonians. For example,

$$
H=\frac{\bar{P}_{\mu} \bar{P}^{\mu}}{2 m}+\frac{1}{2} \alpha \bar{Z}_{\mu \nu} \bar{Z}^{\mu \nu} .
$$

The equations of motion are the same as before except for those of the variables $\theta^{\mu \nu}$, which now satisfy

$$
\dot{\theta}^{\mu \nu}=\frac{1}{2}\left(x^{\mu} \dot{x}^{\nu}-x^{\nu} \dot{x}^{\mu}\right)+\alpha f_{\mu \nu} .
$$

The canonical Lagrangian

$$
\begin{aligned}
L= & \bar{P}_{\mu} \dot{x}^{\mu}+\frac{1}{2} f_{\mu \nu}\left[\dot{\theta}^{\mu \nu}-\frac{1}{2}\left(x^{\mu} \dot{x}^{\nu}-x^{\nu} \dot{x}^{\mu}\right)\right] \\
& -\frac{1}{2} e \bar{P}^{2}-\frac{\alpha}{2} f_{\mu \nu} f^{\mu \nu}
\end{aligned}
$$


gives, after eliminating the nondynamical field $f_{\mu \nu}$, the Lagrangian (6.5).

\section{Other Hamiltonians}

Those which admit a constant $\bar{Z}_{\mu \nu}=F_{\mu \nu}$ and are Lorentz-invariant are of the form

$$
2 m H=\bar{P}_{\mu} \bar{P}^{\mu}+\frac{1}{2} \alpha \bar{Z}_{\mu \nu} \bar{Z}^{\mu \nu}-\beta \bar{Z}_{\mu \nu} \bar{M}^{\mu \nu} .
$$

The second term does not contribute, since it commutes with everything, and so we drop it. Hamilton's equations then give

$$
\dot{\bar{P}}_{\mu}=\frac{1}{m}(1-\beta) F_{\mu \nu} \bar{P}^{\nu}
$$

Note that in the special case $\beta=1$, we find $\dot{\bar{P}}_{\mu}=0$. This is not surprising, because in that case the Hamiltonian is biinvariant; i.e., it is a Casimir, and hence generates no motion at all.

\section{Maxwell-Sim and Maxwell-DISim $b$}

The Maurer-Cartan forms of the coset (Maxwell-Sim)/ (Sim) are the same as in the Maxwell case (2.15), and the moment maps are also given by

$$
\bar{P}_{\mu}=p_{\mu}-\frac{1}{2} f_{\mu \nu} x^{\nu}, \quad \bar{Z}_{\mu \nu}=f_{\mu \nu} .
$$

The geodesic Hamiltonian is given by

$$
H=\frac{\bar{P}_{\mu} \bar{P}^{\mu}}{2 m}+\frac{1}{2} \alpha \bar{Z}_{\mu \nu} \bar{Z}^{\mu \nu},
$$

and therefore reproduces the same dynamics as in the Maxwell case.

For the case of the coset $\left(\right.$ Maxwell-DISim $\left._{b}\right) /(\mathrm{Sim})$, the Maurer-Cartan forms and the momenta are the same as for the Maxwell case.

\section{E. Hamiltonian treatment of the Bogoslovsky-Maxwell algebra}

In previous work [18] we obtained a Finslerian Lagrangian invariant under $\operatorname{DISim}(2)_{b}$, where $b$ is the deformation parameter constructed from the Finslerian line element

$$
d s^{2}=-F\left(v^{\mu}\right)^{2} d \tau^{2},
$$

where the Finsler function $F\left(v^{\mu}\right)$ is homogeneous of degree 1 in the four-velocity $v^{\mu}=d x^{\mu} / d \tau$. In general, if we were to use a multiple of the Finlser function $F\left(v^{\mu}\right)$ as a Lagrangian $L\left(v^{\mu}\right)$, then its Legendre transform would vanish, since a Lagrangian which is homogeneous of degree $k$ in velocities gives, on taking a Legendre transform, a Hamiltonian

$$
\begin{aligned}
H\left(p_{\mu}\right) & =v^{\mu} p_{\mu}-L\left(v^{\mu}\right) \\
& =v^{\mu} \frac{\partial L}{\partial v^{\mu}}-L \\
& =(k-1) L
\end{aligned}
$$

which is homogeneous of degree $\frac{k}{k-1}$ in momenta $p_{\mu}$. If $k=2$ we have

$$
H(p)=L(v)
$$

and both are of degree two. Therefore it is customary in Finsler geometry to set

$$
L\left(v^{\mu}\right)=-\frac{1}{2} m F^{2}\left(v^{\mu}\right)
$$

For the case of Bogoslovsky's Finslerian geometry we would then have

$$
L=-\frac{1}{2} m\left(-n_{\rho} v^{\rho}\right)^{2 b}\left(-\eta_{\mu \nu} v^{\mu} v^{\nu}\right)^{1-b},
$$

where $n^{\mu}=\eta^{\mu \nu} n_{\nu}$ is a constant future-directed null vector. The minus signs appear in (7.44) because $v^{\mu}$ is assumed to be future-directed and timelike. With our signature convention, the inner product $n \cdot v=n_{\mu} v^{\mu}$ is then negative. We find that

$$
\begin{aligned}
p_{\mu}= & b m n_{\mu}(-n \cdot v)^{2 b-1}\left(-v^{2}\right)^{1-b} \\
& +(1-b) m v_{\mu}(-n \cdot v)^{2 b}\left(-v^{2}\right)^{-b},
\end{aligned}
$$

and

$$
H=-\frac{1}{2 m}\left(-\frac{p^{2}}{1-b^{2}}\right)^{1+b}\left(-\frac{n \cdot p}{1-b}\right)^{-2 b} .
$$

Imposing the mass-shell condition $H=-\frac{1}{2} m$, i.e., $F(v)^{2}=1$, leads to Eq. (18) of [18]. In this case, the parameter $\tau$ coincides with the Finslerian measure of proper time along the worldline of the particle. ${ }^{4}$ Equation (7.46) is also equivalent to the expression (6.44) [with $\alpha=$ $-m(1-b)$ and $\left.\beta=-\frac{1}{2} m(1+b)\right]$.

We may also give the expression for $v^{\mu}=\partial H / \partial p_{\mu}$, finding

$$
v^{\mu}=e\left[p^{\mu}-b m\left(-\frac{n^{\nu} p_{\nu}}{m(1-b)}\right)^{((b-1) /(b+1))} n^{\mu}\right]
$$

where

$$
e=-\frac{(1+b) m}{p^{2}}
$$

Again, this is in agreement with the corresponding expression (6.47) obtained in the Lagrangian treatment.

The Lorentz force equation follows from (7.47), and $\dot{\bar{P}}^{\mu}=\left\{\bar{P}^{\mu}, H\right\}$, which implies

$$
\dot{\bar{P}}^{\mu}=-\bar{Z}^{\mu \nu} v_{\nu}
$$

\footnotetext{
${ }^{4} \mathrm{We}$ could instead impose the gauge condition $v^{2}=-1$, but this is less natural in the Finslerian framework.
} 


\section{CONCLUSIONS}

We have constructed the noncentral extensions and deformations of the ISim algebra. The Maxwell-Sim algebra is obtained from the translation generators $\mathbf{P}_{\mu}$ and the noncentral extension $\mathbf{Z}_{\mu \nu}=\left[\mathbf{P}_{\mu}, \mathbf{P}_{\nu}\right]$, together with the $\operatorname{Sim}(n)$ generators $\left(\mathbf{M}_{+i}, \mathbf{M}_{+-}, \mathbf{M}_{i j}\right)$.

In general dimensions, the deformations of MaxwellSim algebra are characterized by two parameters $b$ and $c$. The deformation parametrized by $c$ is the analogue of the $k$ deformation of the Maxwell algebra found in [25], which gave $S O(3,2) \times S O(3,1)$ or $S O(4,1) \times S O(3,1)$, depending on the sign of $k$. The $b$ deformation of Maxwell-Sim produces the Maxwell extension of the $\mathrm{DISim}_{b}$ algebra, which is related to Finslerian geometry.

We have also studied the motion of a massive particle interacting with a constant electromagnetic field with these symmetries. In the case of Maxwell-DISim $b$, the motion is given by a Finslerian Lorentz force, while by contrast for the undeformed Maxwell-Sim algebra we obtain the ordinary Lorentz force.

\section{ACKNOWLEDGMENTS}

We acknowledge discussions with Sotirios Bonanos, Roberto Casalbuoni, Kiyoshi Kamimura, David Kubiznak, and Mikhail Vasiliev. This work was initiated at the Galileo Galilei Institute in Florence, continued at the Benasque School, at CTC in DAMTP, and concluded in the Department of Physics in Barcelona. The authors would like to thank those institutions for their hospitality and support. We also acknowledge financial support from Projects No. FPA2007-66665-C02-01, No. 2009SGR502, and Consolider CPAN CSD2007-00042. The work of C.N.P. is supported in part by DOE Grant No. DEFG03-95ER40917.

\section{APPENDIX A: CONVENTIONS}

In this appendix we record some of our conventions and notation when working with Lie groups, Lie algebras, and Poisson algebras.

Given a Lie group $G$, with coordinates $x^{\mu}$, i.e., group elements $G \ni g=g\left(x^{\mu}\right)$, and left- and right-invariant Cartan-Maurer forms

$$
g^{-1} d g=\lambda^{a} \mathbf{e}_{a}, \quad d g g^{-1}=\rho^{a} \mathbf{e}_{a},
$$

with $\mathbf{e}_{a}$ a basis for the Lie algebra $g$ such that

$$
\left[\mathbf{e}_{a}, \mathbf{e}_{b}\right]=C_{a}{ }_{b}^{c} \mathbf{e}_{c},
$$

the Maurer-Cartan equations are

$$
d \lambda^{c}=-\frac{1}{2} C_{a}{ }_{b}{ }^{a} \lambda^{a} \wedge \lambda^{b}, \quad d \rho^{c}=\frac{1}{2} C_{a}{ }^{c}{ }_{b} \rho^{a} \wedge \rho^{b} .
$$

The left- and right-invariant vector fields $L_{a}^{\mu}$ and $R_{a}^{\mu}$ dual to $\lambda_{\mu}^{a}$ and $\rho_{\mu}^{a}$, respectively,

$$
\lambda_{\mu}^{a} L_{b}^{\mu}=\delta_{b}^{a}, \quad \rho_{\mu}^{a} R_{b}^{\mu}=\delta_{b}^{a},
$$

satisfy

$$
\begin{aligned}
& {\left[L_{a}, L_{b}\right]=C_{a}{ }^{c}{ }_{b} L_{c} \quad\left[R_{a}, L_{b}\right]=0,} \\
& {\left[R_{a}, R_{b}\right]=-C_{a}{ }^{c}{ }_{b} R_{c},}
\end{aligned}
$$

and, respectively, generate right and left translations on $G$.

Quantum mechanically, one often inserts $i$ 's so that if $\hat{R}_{a}=\frac{1}{i} R_{a}, \hat{L}_{a}=\frac{1}{i} L_{a}$ then

$$
\begin{gathered}
{\left[\hat{R}_{a}, \hat{R}_{b}\right]=i C_{a}{ }^{c}{ }_{b} \hat{R}_{c},} \\
{\left[\hat{L}_{a}, \hat{L}_{b}\right]=-i C_{a}{ }^{c}{ }_{b} \hat{L}_{c} .}
\end{gathered}
$$

The $\hat{R}_{a}$ and $\hat{L}_{a}$ vector fields are then operators acting on complex-valued functions of the group coordinates $x^{\mu}$.

Thinking of $G$ as a configuration space, we can pass to the phase space or cotangent space $T^{G^{\star}} \equiv G \times \mathfrak{g}$, with coordinates $\left(x^{\mu}, p_{\nu}\right)$. The actions of $G$ on $G$ then lift to $T^{G^{\star}}$ as canonical transformations, leaving the natural symplectic form $d p_{\mu} \wedge d x^{\mu}$ invariant. Given the symplectic form, we can introduce the Poisson bracket as usual. In local Darboux coordinates $\left(x^{\mu}, p_{\nu}\right)$, it is given by

$$
\{f, g\}=\frac{\partial f}{\partial x^{\mu}} \frac{\partial g}{\partial p_{\mu}}-\frac{\partial g}{\partial x^{\mu}} \frac{\partial f}{\partial p_{\mu}},
$$

so that

$$
\left\{x^{\mu}, p_{\nu}\right\}=\delta_{\nu}^{\mu} .
$$

Infinitesimally, the lifts of left and right actions are canonical transformations generated by "generating functions" or "moment maps." Because, in general, we have both left and right actions to take into account, we define two sets of moment maps into $\mathrm{g}^{\star}$, the dual of the Lie algebra,

$$
M_{a}=p_{\mu} L_{a}^{\mu}, \quad N_{a}=p_{\mu} R_{a}^{\mu},
$$

with Poisson brackets which are readily seen to be

$$
\begin{aligned}
& \left\{M_{a}, M_{b}\right\}=-C_{a}{ }^{b}{ }_{c} M_{b}, \quad\left\{M_{a}, N_{b}\right\}=0, \\
& \left\{N_{a}, N_{b}\right\}=C_{a}{ }^{b}{ }_{c} N_{b} .
\end{aligned}
$$

The moment maps $M_{a}$ generate the lifts of right translations and the moment maps $N_{a}$ generate the lifts of left translations.

A Hamiltonian $H=H\left(x^{\mu}, p_{\mu}\right)$, which is left-invariant, satisfies

$$
\dot{N}_{a}=\left\{N_{a}, H\right\}=0,
$$

and so the moment maps $N_{a}$ are constants of the motion. By contrast, the moment maps $M_{a}$ generating right actions are time-dependent,

$$
\dot{M}_{a}=\left\{M_{a}, H\right\} \neq 0 .
$$


A left-invariant Lagrangian may be constructed from combinations of left-invariant velocities or angular velocities

$$
\omega^{a}=\lambda_{\mu}^{a} \dot{x}^{\mu} .
$$

Thus the Hamiltonian is a combination of the momenta maps $M_{a}$,

$$
H=H\left(M_{a}\right) .
$$

Thus (A13) provides an autonomous first-order system of ordinary differential equations on $\mathrm{g}^{\star}$ for the moment maps $M_{a}$, called the Euler equations. To obtain the motion on the group, one uses the equation

$$
\dot{x}^{\mu}=\frac{\partial H}{\partial p_{\mu}} .
$$

Now

$$
p_{\mu}=M_{a} \lambda_{\mu}^{a},
$$

and so

$$
\dot{x}^{\mu}=L_{a}^{\mu} \frac{\partial H}{\partial M_{a}} .
$$

\section{APPENDIX B: LIFSHITZ AND SCHRÖDINGER ALGEBRAS}

In this appendix we shall describe the connection between the deformed inhomogeneous Sim algebra $\operatorname{Digim}_{b}(k)$ and the Lifshitz, Schrödinger, and extended Schrödinger algebras, $\mathfrak{l i f}_{z}, \mathfrak{s c h}_{z}(k)$, and $\widetilde{\mathfrak{s c h}}(k)$, respectively.

\section{Lifshitz scaling}

In nonrelativistic theories with $k$ spatial dimensions, one is interested in the behavior of physical quantities under what has come to be called Lifshitz scaling, i.e., under

$$
t \rightarrow \lambda^{z} t, \quad \mathbf{x} \rightarrow \lambda \mathbf{x}
$$

where $t$ is the time variable and $\mathbf{x}=\left(x_{1}, x_{2}, \ldots, x_{k}\right)$ is the spatial position vector.

If $D$ generates scalings or dilatations, we may combine this with space translations $P_{i}$, spatial rotations $M_{i j}$, and time translations $H$ to obtain the Lifshitz algebra, $\operatorname{lif}_{z}(k)$, in $k$ spatial dimensions,

$$
\left[D, M_{i j}\right]=0, \quad\left[D, P_{i}\right]=P_{i}, \quad[D, H]=z H,
$$

where the obvious brackets for $M_{i j}$ have been omitted. The Lie algebra spanned by $D, P_{i}$, and $H$ is therefore invariant under the adjoint action of the rotation subalgebra $\mathfrak{S D}(k)$ generated by $M_{i j}$. If $i=1,2, \ldots, k$, then $\mathfrak{I i f}_{z}(k)$ has dimension $\frac{1}{2} k(k+1)+2$ and the quotient $\mathfrak{l i f}_{z}(k) / \mathfrak{s} \mathfrak{D}(k)$ has dimension $k+2$.

\section{Lifshitz spacetime}

This is a $(k+2)$-dimensional spacetime equipped with a metric invariant under the left action of the $(k+2)$ dimensional group generated by $P_{i}, H$, and $D$. A Maurer-Cartan basis for this solvable group is

$$
e^{r}=\frac{d r}{r}, \quad e^{i}=\frac{d x^{i}}{r}, \quad e^{0}=\frac{d t}{r^{z}} .
$$

The Lifshitz metric is then

$$
d s_{k+2}^{2}=L^{2}\left\{-\frac{d t^{2}}{r^{2 z}}+\frac{d x_{i} d x_{i}}{r^{2}}+\frac{d r^{2}}{r^{2}}\right\},
$$

with Killing vector fields corresponding to

$$
\begin{aligned}
& M_{i j}=-\left(x_{i} \partial_{j}-x_{j} \partial_{i}\right), \quad P_{i}=-\partial_{i}, \quad H=-\partial_{t}, \\
& D=-\left(z t \partial_{t}+x_{i} \partial_{i}+r \partial_{r}\right) .
\end{aligned}
$$

(i) As $r \rightarrow \infty$ we approach a singular horizon (IR limit).

(ii) As $r \rightarrow 0$ we approach infinity (UV limit).

The boundary metric at infinity is obtained by taking out a factor of $r^{2}$ and letting $r \rightarrow 0$ :

$$
d s_{k+2}^{2}=\frac{L^{2}}{r^{2}}\left\{-\frac{d t^{2}}{r^{2(z-1)}}+d x_{i} d x_{i}+d r^{2}\right\} .
$$

Thus

$$
d s_{\text {boundary }}^{2}=d x_{i} d x_{i}-r^{2(1-z)} d t^{2},
$$

the speed is $c(r)=r^{(1-z)}$, and

(i) If $z>1$, we obtain infinite speed (the boundary light cone opens out to a plane).

(ii) If $z=1$, we obtain finite speed (the boundary light cone remains a cone).

(iii) If $z<1$, we obtain zero speed (the boundary light cone closes up to a half line).

Strictly speaking, in the $z>1$ case, we need to consider the inverse metric when taking the limit $r \rightarrow 0$.

\section{The boost-extended Lifshitz algebra}

One may extend the Lifshitz algebra to include boosts $K_{i}$. The scaling dependence of $K_{i}$ is then determined by its commutation relations. Since $K_{i}$ is a vector, we have

$$
\left[K_{k}, M_{i j}\right]=-\left(\delta_{k i} K_{j}-\delta_{k j} K_{i}\right) .
$$

For the Galilei group,

$$
\begin{aligned}
& {\left[K_{i}, P_{j}\right]=0,} \\
& {\left[K_{i}, H\right]=P_{i},}
\end{aligned}
$$

which implies that we must take

$$
\left[D, K_{i}\right]=(1-z) K_{i} .
$$


For the Carroll group

$$
\begin{gathered}
{\left[K_{i}, P_{j}\right]=\delta_{i j} H,} \\
{\left[K_{i} H\right]=0,}
\end{gathered}
$$

which implies that we must take

$$
\left[D, K_{i}\right]=(z-1) K_{i} \text {. }
$$

In the case of the Poincare group there is no choice, and one must take $z=1$.

\section{4. $\operatorname{DISim}_{b}(k)$}

Recall that $\operatorname{DISim}_{b}(k)$ is a deformation of the $\operatorname{ISim}(k)$ subgroup of the Poincaré group in $(k+2)$ spacetime dimensions, depending on a parameter $b$, which may be regarded as a subgroup of the inhomogeneous Weyl group or causal group (i.e., the semidirect product of Poincaré with dilatations), in which the actions of a boost and dilations are identified up to a factor [18]. It is thus of dimension $\frac{1}{2} k(k+1)+k+3$.

If the translations are $P_{+}, P_{-}, P_{i}$, and the boosts are $M_{+i}, M_{+-}$, then the nontrivial Lie brackets are given by

$$
\begin{aligned}
& {\left[M_{+-}, P_{ \pm}\right]=-(b \pm 1) P_{ \pm}, \quad\left[M_{+-}, P_{i}\right]=-b P_{i},} \\
& {\left[M_{+-}, M_{+i}\right]=-M_{+i}, \quad\left[M_{+i}, P_{-}\right]=P_{i},} \\
& {\left[M_{+i}, P_{j}\right]=-\delta_{i j} P_{+} .}
\end{aligned}
$$

The $\mathfrak{S D}(k)$ rotations have the standard brackets and act on $P_{i}$ and $M_{+i}$ as vectors. The boost generator $M_{+-}$acts on $(k+2)$-dimensional Minkowski spacetime as

$$
x^{i} \rightarrow \lambda^{-b} x^{i}, \quad x^{-} \rightarrow \lambda^{1-b} x^{-}, \quad x^{+} \rightarrow \lambda^{-1-b} x^{+} .
$$

If $b=0$, then $M_{+-}$acts as an ordinary boost.

\section{The Schrödinger and extended Schrödinger algebras}

In $k$ spatial dimensions, the centrally extended $\left[\frac{1}{2} k(k+1)+k+3\right]$-dimensional Schrödinger algebra (in current terminology [22]), which we denote $\widetilde{\mathfrak{F C h}_{z}}(k)$, is obtained by adjoining Galilean boosts $K_{i}$, and a central term $N$, to the Aristotelian algebra of translations, rotations, and time translations, such that

$$
\begin{gathered}
{\left[M_{i j}, K_{k}\right]=\left(\delta_{i k} K_{j}-\delta_{j k} K_{i}\right),} \\
{\left[P_{i}, K_{j}\right]=-\delta_{i j} N,} \\
{\left[H, K_{i}\right]=-P_{i} .}
\end{gathered}
$$

The result is the $\left[\frac{1}{2} k(k+1)+k+2\right]$-dimensional Bargmann algebra, a central extension of the $\left[\frac{1}{2} k(k+1)+\right.$ $k+1$ ]-dimensional Galilei algebra. One then adjoins a dilatation $D$,

$$
\left[D, K_{i}\right]=(1-z) K_{i}, \quad[D, N]=(2-z) N .
$$

If $k=3$ this is 12 -dimensional, whereas what has been called the Schrödinger group, i.e., the conformal symmetry group of the free Schrödinger equation (corresponding to $z=2$ ), is 13 -dimensional. ${ }^{5}$ This is because the special conformal or temporal inversion operator has been left out.

One may consistently drop the central extension $N$ from the Bargmann algebra to get the Galilei algebra, and then the extended Schrödinger algebra $\widetilde{\mathfrak{s c h}}(k)$ reduces to the $\left[\frac{1}{2} k(k+1)+k+2\right]$-dimensional unextended Schrödinger algebra $\mathfrak{s} \mathfrak{c h}(k)$. If one then drops the boost generator $K_{i}$ one gets the Lifshitz algebra $\operatorname{lif}_{z}(k)$.

It is well known that nonrelativistic symmetries and nonrelativistic conformal symmetries (Schrödinger algebras) in $k$ spatial dimensions may be thought of as subgroups of relativistic or conformal symmetries in $(k+2)$-dimensional Minkowski spacetime which commute with lightlike translations. Thus it is no surprise that

$$
\widetilde{\mathfrak{s c h}}_{z}(k) \equiv \operatorname{Disim}_{b}(k), \quad b=\frac{1}{1-z} .
$$

To see this, one must identify the generators as follows:

$$
H \leftrightarrow P_{-}, \quad N \leftrightarrow-P_{+}, \quad P_{i} \leftrightarrow P_{i}, \quad K_{i} \leftrightarrow M_{+i},
$$

and

$$
D \leftrightarrow(z-1) M_{+-} .
$$

Note that it is also possible to obtain the Lifshitz algebra $\mathfrak{I f f}(k)$ as a truncation of the $\operatorname{Digimm}_{b}(k)$ algebra by discarding the $P_{i}$ generators and making the identifications

$$
H \leftrightarrow P_{-}, \quad P_{i} \leftrightarrow M_{+i}, \quad D \leftrightarrow M_{+-},
$$

and

$$
z=(b-1) .
$$

However, this is perhaps less useful than the identification (B20).

\section{Schrödinger spacetime}

This is $(k+3)$-dimensional, and has metric

$$
d s_{k+3}^{2}=L^{2}\left\{-\frac{d t^{2}}{r^{2 z}}-\frac{2 d t d v}{r^{2}}+\frac{d x_{i} d x_{i}}{r^{2}}+\frac{d r^{2}}{r^{2}}\right\}
$$

with Killing vectors

$$
\begin{gathered}
K_{i}=-\left(t \partial_{t}+x^{i} \partial_{v}\right), \quad N=-\partial_{v}, \\
P_{i}=-\partial_{i}, \quad M_{i j}=-\left(x_{i} \partial_{j}-x_{j} \partial_{i}\right), \\
D=-\left(z t \partial_{t}+x_{i} \partial_{i}+(2-z) v \partial_{v}+r \partial_{r}\right) .
\end{gathered}
$$

\footnotetext{
${ }^{5}$ The reader should note the difference with the Galilean conformal algebra obtained by contraction from the relativistic conformal algebra, which has 15 generators (see, for example, $[39,40])$.
} 
A Cartan-Maurer basis for this solvable group manifold is given by

$$
e^{r}=d r / r, \quad e^{i}=\frac{d x^{i}}{r}, \quad e^{v}=\frac{d v}{r^{2-z}}, \quad e^{t}=\frac{d t}{r^{z}} .
$$

\section{Lifshitz spacetime as a null reduction of Schrödinger spacetime}

If we identify points in the Schrödinger spacetime under the $\mathbb{R}$ action generated by the null Killing field $\partial_{v}$, i.e., under the action of the "central" element $N$, we obtain the Lifshitz spacetime. On the boundary we have the metric

$$
d s_{\text {boundary }}^{2}=d x_{i} d x_{i}-2 d t d v-r^{2(1-z)} d t^{2} .
$$

In the cases $z>1$, we may regard the boundary as the $(k+2)$-dimensional Duval-Kunzle spacetime whose null reduction produces the $(k+1)$-dimensional NewtonCartan spacetime. Strictly speaking we need to consider the inverse metric when taking the limit.
[1] A. Lees, Philos. Mag. Series 7 28, 385 (1939).

[2] P. A. M. Dirac, Proc. R. Soc. A 268, 57 (1962).

[3] Y. Nambu, "Duality and Hadrodynamics" Lectures at the Copenhagen Summer Symposium" (1970). Reprinted in Broken Symmetry: Selected Papers of Y. Nambu, edited by T. Eguchi and K. Nishijima (World Scientific, Singapore, 1995).

[4] T. Goto, Prog. Theor. Phys. 46, 1560 (1971).

[5] J. Hughes and J. Polchinski, Nucl. Phys. B278, 147 (1986).

[6] A. S. Schwarz, J. Math. Phys. (N.Y.) 51, 015201 (2010).

[7] J. P. Gauntlett, K. Itoh, and P. K. Townsend, Phys. Lett. B 238, 65 (1990).

[8] J. Gomis, K. Kamimura, and P. C. West, Classical Quantum Gravity 23, 7369 (2006).

[9] E. A. Ivanov and V. I. Ogievetsky, Teor. Mat. Fiz. 25, 164 (1975).

[10] H. von Helmholtz, On the Origin and Significance of Geometrical Axioms (1870) [translated in Popular Lectures, reprinted by Dover], also available as Mind os1, 301 (1876).

[11] J.H. Lambert, Theorie der Paraliellinien, as quoted in D. M. Y. Sommerville, The Elements of Non-Euclidean Geometry (1914), reprinted by Dover.

[12] A. Calinon, Revue Philosophique 36, 595 (1893).

[13] E. İnönü and E. P. Wigner, Proc. Natl. Acad. Sci. U.S.A. 39, 510 (1953).

[14] A. Einstein, in Sitzungsberichte der Preussischen Akademie der Wissenschaften (Berlin, 1917), p. 142.

[15] W. de Sitter, Koninklijke Nederlandsche Akademie van Wetenschappen, Proceedings (1917), Vol. 19, p. 1217.

[16] H. Bacry and J. Levy-Leblond, J. Math. Phys. (N.Y.) 9, 1605 (1968).

[17] A. G. Cohen and S. L. Glashow, Phys. Rev. Lett. 97, 021601 (2006).

[18] G. W. Gibbons, J. Gomis, and C. N. Pope, Phys. Rev. D 76, 081701 (2007).

[19] G. Y. Bogoslovsky, in Proc. Int. Conf. "Physical Interpretations of Relativity Theory" (to be published).

[20] G. W. Gibbons, J. Gomis, K. Kamimura, and C. N. Pope (unpublished) [see, e.g., G.W.G., "Very Special Relativity and Non-Relativistic Symmetries" at Atelier:
Symétries Non Relativistes, University of Tours, June 2009] and independently by A. Davody and H. R. Afshar (private communication).

[21] G. Burdet, M. Perrin, and P. Sorba, Commun. Math. Phys. 34, 85 (1973).

[22] K. Balasubramanian and J. McGreevey, Phys. Rev. Lett. 101, 061601 (2008).

[23] A. A. Abdo et al. (Fermi GBM/LAT Collaborations), Nature 462, 331 (2009).

[24] J. W. van Holten and A. Van Proeyen, J. Phys. A 15, 3763 (1982).

[25] J. Gomis, K. Kamimura, and J. Lukierski, J. High Energy Phys. 08 (2009) 039.

[26] S. R. Coleman, J. Wess, and B. Zumino, Phys. Rev. 177, 2239 (1969); C. G. Callan, S. R. Coleman, J. Wess, and B. Zumino, Phys. Rev. 177, 2247 (1969).

[27] E. M. Stein, in High Energy Physics and Elementary Particles (International Atomic Energy Agency, Vienna, 1965).

[28] R. Schrader, Fortschr. Phys. 20, 701 (1972).

[29] H. Bacry, Ph. Combe, and J. L. Richard, Il Nuovo Cimento A 67, 267 (1970).

[30] H. Bacry, Ph. Combe, and J. L. Richard, Il Nuovo Cimento A 70, 289 (1970).

[31] A. Janner and E. Ascher, Physica (Amsterdam) 48, 425 (1970).

[32] Ph. Combe and J. L. Richard, Physica (Amsterdam) 71, 66 (1974).

[33] H. Hoogland, J. Phys. A 11, 797 (1978).

[34] D. V. Soroka and V. A. Soroka, Adv. High Energy Phys. 2009, 234147 (2009).

[35] S. Bonanos and J. Gomis, J. Phys. A 42, 145206 (2009).

[36] M. Levy-Nahas, J. Math. Phys. (N.Y.) 8, 1211 (1967).

[37] S. Bonanos, "Exterior Differential Calculus," Mathematica Enhancement, http://www.inp.demokritos .gr/sbonano/EDC/.

[38] S. Bonanos and J. Gomis, J. Phys. A 43, 015201 (2010).

[39] J. Negro, M. A. del OLmo, and A. Rodriguez-Marco, J. Math. Phys. (N.Y.) 38, 3786 (1997).

[40] J. Lukierski, P. C. Stichel, and W. J. Zakrzewski, Phys. Lett. A 357, 1 (2006). 